\title{
DEVELOPMENT OF SEMICONDUCTOR AND SUPERCONDUCTOR TECHNOLOGY AND FUTURE BIOSCIENCE
}

\author{
TING-TANg LAI ${ }^{1}$, HoNG-SHENG LEE ${ }^{2}$ \\ ${ }^{1}$ Department of Otolaryngology, Wang-Feng Hospital \\ Taipei Medical University \\ ${ }^{2}$ En Chu Kong Hospital, Prof. Emeritus of Taipei Medical University
}

\section{INTRODUCTION}

The biosciences have rushed into a new era since international efforts to analyze the human genome were completed at the end of the 20th century. This remarkable achievement, along with modern advances in biotechnology, has given birth to the fields of gene diagnosis and gene therapy, and a series of other biosciences with new names such as pharmacogenetics, pharmacogenomics, proteomics, bioinformatics, nano biotechnology, etc.

These sciences are all linked by a basic philosophy and common technology, collectively achieving a new scope of medical treatments using BioMEMS (Bio/Micro/Electro/Mechanical System)[1]. And since the current trend in science is to progress from the micro-world to the nano-world, applications of MEMS are gradually being replaced by NEMS, as in BioNEMS (Bio/Nano/Electro/Mechanical System) [2] (Table. 1).

The new generation of biosciences requires technologies that are capable of analyzing and applying large volumes of data, collecting specific information on individual tissues, organs, or bodies, and building precision sensors that are highly sensitive and miniature in size. These complex technologies can only be achieved through dramatic advances in computer systems, discoveries of new biosensors, and the development of new biomaterials. This presentation will outline the ongoing developments in semiconductors and superconductivity for bioscientific applications (Fig. 1).

Received: Sep 8, 2004; Accepted: Sep 15, 2004

Correspondence: Hong-Sheng Lee, Professor

En Chu Kong Hospital, Prof.

Emeritus of Taipei Medical University

E-mail: 10487@km.eck.org.tw
Table 1. RECENT PROGRESS IN BIOSCIENCE

1. Pharmacogenomics

2. Pharmacogenetics

3. Proteomics

4. Bioinformatics

5. BioMEMS (Bio/Micro/Electronics/Mechanical System) or BioNEMS (Bio/Nano-System)

6. Nanobiotechnology

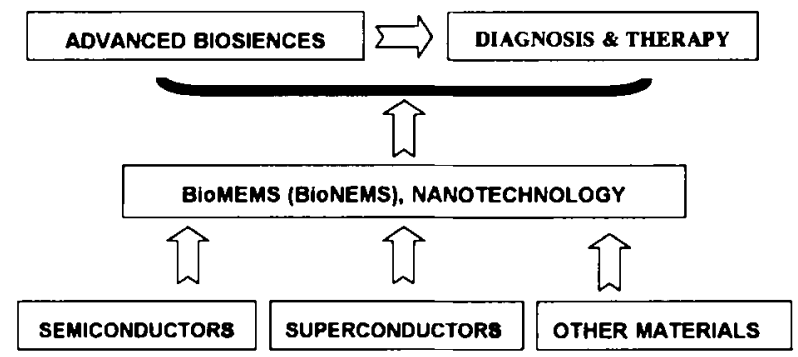

Fig. 1 Advanced Diagnosis \& Therapy in near Future

\section{DISCOVERY AND FUTURE SEMICONDUCTORS[3]}

As the name indicates, semiconductors are materials with electrical conductivities that are intermediate between those of conductors and insulators. The two most common elemental semiconductors are germanium $(\mathrm{Ge})$, which has limited practical use due to inherent physical and chemical problems, and silicon (Si). Silicon exists abundantly on the earth and has become the element of choice in making transistors. 
Pure silicon is a very stable crystal having four valence electrons in the outermost orbit, each of which is covalently bonded with one of four adjacent silicon atoms so that almost all of the electrons are constrained. Therefore, silicon atoms in their pure form are impractical for conduction purposes because semiconductors require free electrons or electron hole propagation to carry electric currents.

Nonetheless, it is well known that impurity semiconductors prepared by incorporating a foreign atom ( "impurity") into the crystal lattice of a silicon atom have improved control over electric conductivity. The impurity can be an atom with three valence electrons such as boron (B), or five valence electrons such as phosphorus (P), arsenic (As), or antimony (Sb) (Fig. 2).

When a trivalent impurity element such as boron is substituted into the silicon crystal lattice, an electron is missing from one of the four possible covalent bonds. The atom can therefore accept an electron to complete the fourth bond, resulting in the subsequent formation of a hole (hole propagation). This type of impurity semiconductor is called a "p-type semiconductor." On the other hand, if an atom with five valence electrons such as phosphorus or arsenic is incorporated into the crystal lattice of a silicon atom, the impurity atom has four covalent bonds and one unbonded free electron, and this free electron is the key to inducing conductivity. This type of impurity semiconductor is called an "n-type semiconductor" (Fig. 3).

P-type and n-type semiconductors can be connected to form a diode, which is a type of transistor that allows electric current to flow in only one direction. If a positive bias voltage is placed on the $p$ type side, the holes (dominant positive carriers) are pushed toward the junction. At the same time, the free electrons (dominant negative carriers) on the ntype side are attracted toward the junction. If the amounts of these carriers are scarce, the electric charges of these carriers would be lost by the collision at the junction and consequently current does not flow. But in fact, there is an abundance of carriers at the junction and current can indeed flow across the junction (Fig. 4A). However, if the bias is reversed,

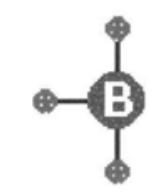

BORON TRIVALENT

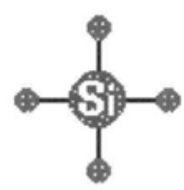

SILICON TETRAVALENT

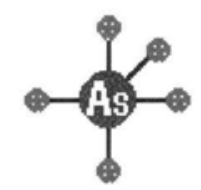

ARSENIC PENTAVALENT
Fig. 2 Impurity atoms incorporated into silicon[4] the holes and electrons are pulled away from the junction, leaving the junction as a depletion layer that prevents current flow (Fig. 4B).

Most transistors are bipolar and consist of three units, that is, $n-p-n$ or $p-n-p$ type semiconductors. Bipolar transistors having three terminals are used for electric amplification (Fig. 5), and the function of transistors can be increased remarkably by developing a device in which the field effect produced through an insulating layer is used. This type of transistor, a metal oxide - field effect transistor (MOSFET) performs the central role of transistor systems (Fig. 6).

The integrated circuit (IC) was developed in 1959 as a revolutionary new electrical element consisting of transistors, resistors, and condensers arranged on a small silicon tip. IC development has since then accelerated exponentially thanks to advanced techniques for miniaturizing transistors. At present, the industry's developments have produced LSI (largescale integrated circuits containing 1,000 to 10,000
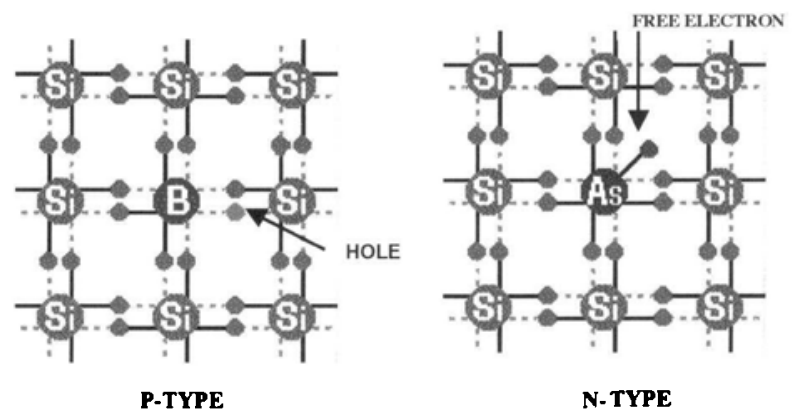

Fig. 3 Impurity semiconductors: P- AND NTYPE[5-6]

A
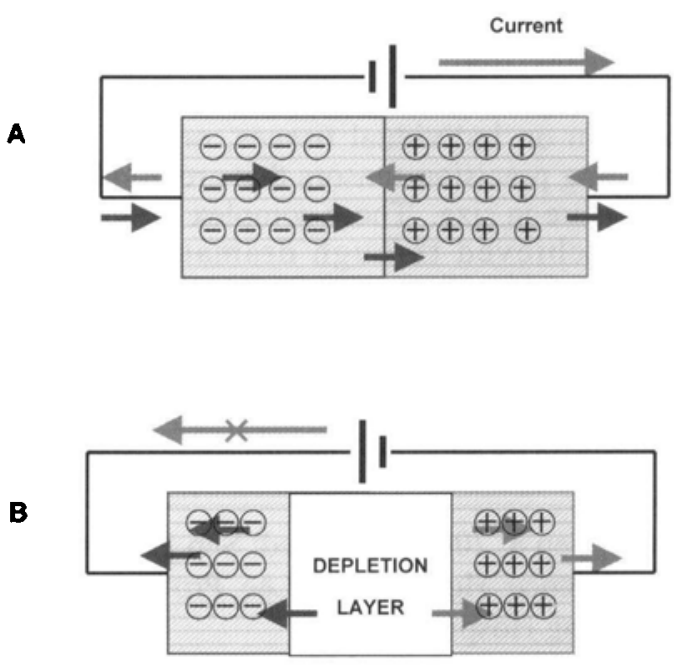

Fig. 4 Semiconductor diode with Pn- junction 


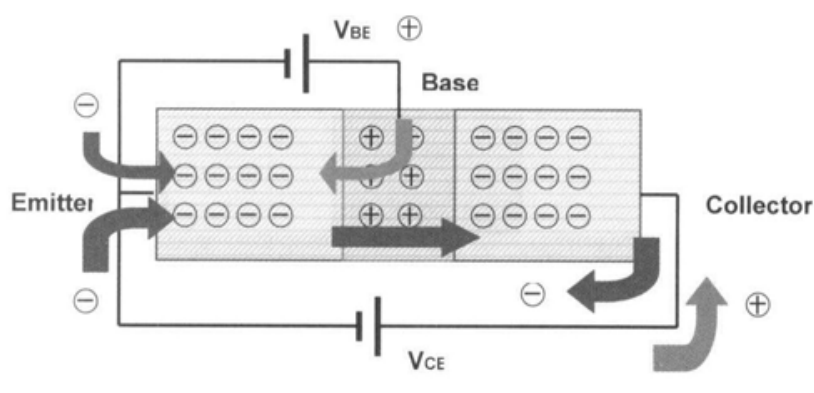

Fig. 5 N-P-N type bipolar transistor

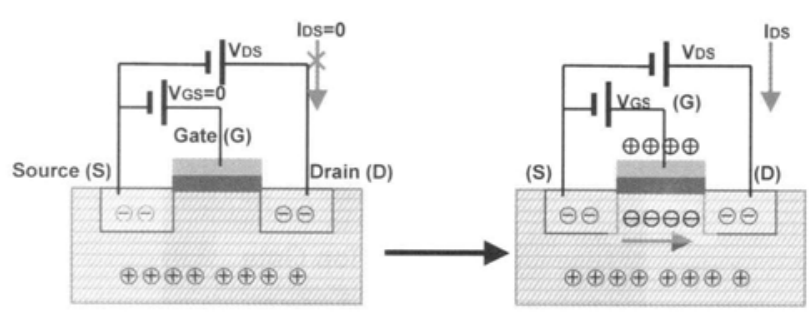

Fig. 6 Mosfet (metal oxide - field effect transistor)

transistors), VLSI (very large-scale integrated circuits containing more than 100,000 transistors), and ULSI (ultra large-scale integrated circuits containing more than one million transistors).

The progress of IC technology has so far fulfilled the 1965 prediction made by Dr. Gordon Moore, cofounder of Intel Corp. Moore' s law states, "the number of transistors on a given chip can be doubled every two years." (Fig. 7).

While Moore' $s$ law has generally been accepted up to the present, it may not be applicable by the year 2010 due to various limitations on existing technology. To conquer these problems, further miniaturization of transistors and other electric elements is required, along with the development of high-performance ICs.

For its part, the International Technology Roadmap for Semiconductors (ITRS)[8] sponsors annual conferences for the semiconductor community from the U.S., E.U., Japan, Taiwan and South Korea. ITRS meetings address issues of setting target values for minimizing transistors and finding technical developments required to attain these target values.

In the 1970s at the dawn of semiconductor computer history, scientists were able to load only about 4,000 electric elements onto a tip. Nowadays, scientists can easily load up to a billion $\left(1 \mathrm{G}\right.$, or $\left.10^{9}\right)$ pieces of elements.

A scale of $256 \mathrm{MB}$ is common capacity for Random Access Memory (RAM: freely accessible) in an ordinary personal computer. Further advances in

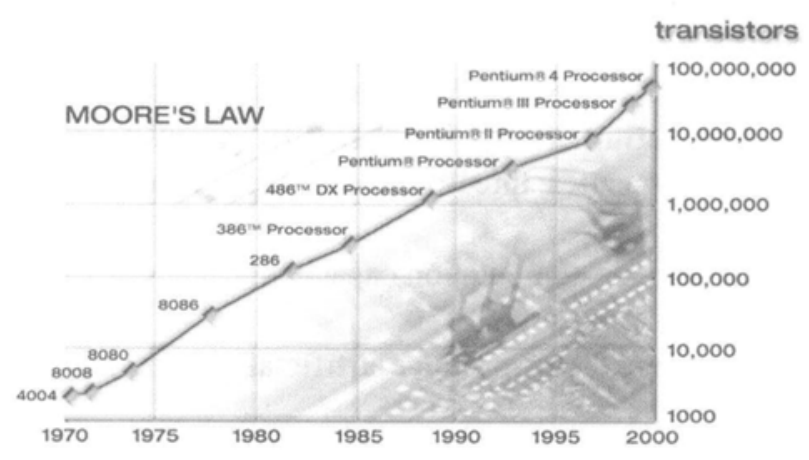

Fig. 7 Moore's law: exponential groeth in the number of transistors per integrated circuit 7

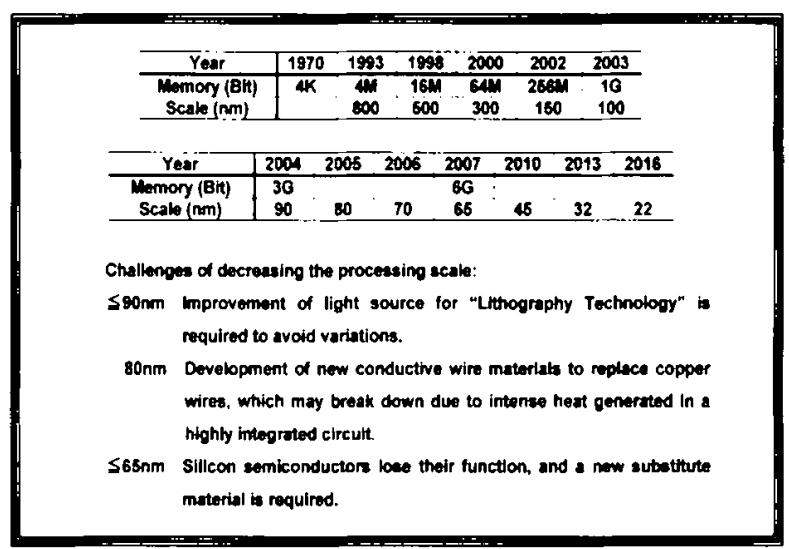

Fig. 8 Increasing memory capacity VS. decreasing processing scale

the miniaturization of transistors promise denser loading of electric elements, which can bring about remarkable increases in memory capacity, computing power, new functionality, etc. (Table 2).

However, there are a large number of scientific problems that must be overcome in order to achieve these target values. Why should we commit such enormous efforts to attaining these goals? What kinds of needs are there for advancing semiconductor sciences?

The new biosciences alone present important demands for high-performance computing. For instance, there are numerous needs for the prompt transmission and reception of mass databases such as the exchange of new gene information, development of miniaturized BioNEMS materials/apparatus for diagnosis/therapy, application of virtual reality information, solution of Hamilton course problems against which our conventional von Neumann type computers are inadequate, analysis of three- 
Table 2 Future goals of transitor development

To transmit/receive large volumes of data such as gene information, virtual reality information for bioinformatics, three-dimensional proteomics, etc.

To upgrade computation capacity

To explore and produce artificial brains, robots, etc.

dimensional structures of protein/enzyme indispensable for proteomics and new drugs, and ultimately, the development of artificial intelligence that exceeds the human brain in function.

However, even if transistors continue to shrink to miniscule dimensions, they still retain the same structure and principle as that of first MOSFET invented in 1974. That is, a voltage applied to a gate electrode in the middle position controls electron flows from a source electrode to a drain electrode, and operates functions from on-off switching to amplification. Since a gate length determines the distance from a source to a drain and also serves as a limiting factor in miniaturization, it is necessary to make the gate length as small as possible. Presently, the minimum gate length used in transistors is $70 \mathrm{~nm}$, and an experimental transistor with gate length as thin as $6 \mathrm{~nm}$ has also been reported[9]. Since the distance between silicon atoms is approximately $0.3 \mathrm{~nm}$, a gate length approaching this size will only allow a few electrons to flow between source and drain electrodes. In this situation, the fundamental field effect principle of MOSFETs is no longer demonstrated. Furthermore, if the MOSFET principle is inherited and miniaturization continues, problems of current leakage or generation of heat accompanying overcrowded structures will come to the fore. Therefore, researchers are searching for a new science that can exceed the capabilities of present silicon semiconductors (Fig. 9).

Development of transistors with two or three gates, i.e., double-gate[10] or tri-gate[11] transistors, has been explored as a means of keeping Moore's law while attaining ultimate miniaturization. The problems of current leakage accompanying miniaturization can be avoided for a time by means of these types of transistors (Fig. 10).

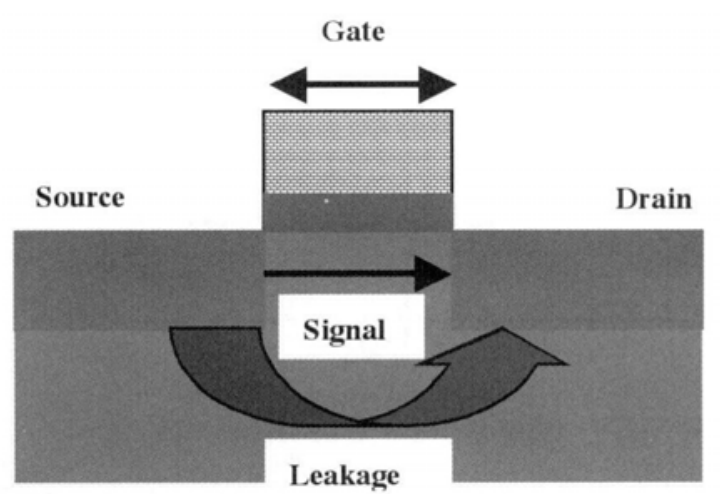

Fig. 9 Relation between "gate length" and "leakage current" in mosfet
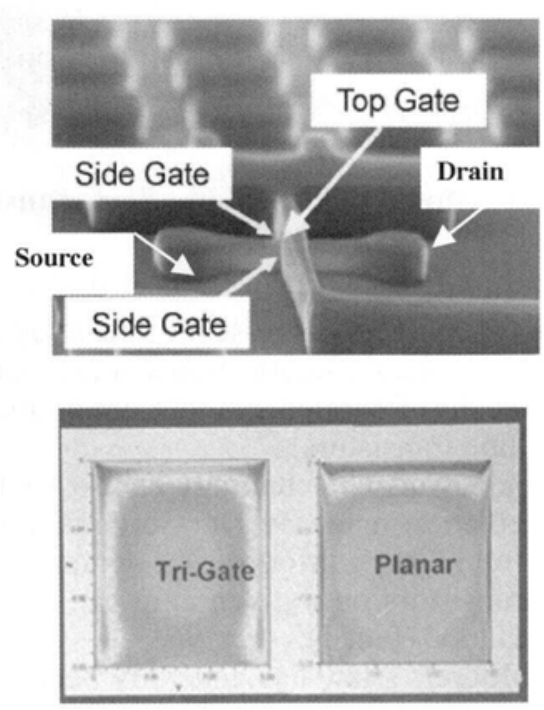

Fig. 10 Tri-gate transistor(intel)

\section{SEMICONDUCTOR BIO- DEVICES, BIOMEMS}

It is clear that future progress in medical treatments will drive demands for high performance computers, along with needs for more improvements in semiconductor technology. Apart from meeting the demands of future computers, semiconductor technology can also be applied towards inventing various minute machine systems called Micro ElectroMechanical Systems (MEMS). MEMS has been applied to a wide range of natural sciences, including information technology, automobiles, the environment, 
medicine, biology, architecture, examination/inspection, etc. This paper will specifically describe BioMEMS, the system relating to medicine and biology.

Technically, the system incorporates electronic circuits, chemical reaction cells, a control system, analysis apparatus, and machine parts onto a thin silicon tip of several square millimeters, and then applies this tip to medical treatment. There are systems for extra and intracorporeal uses.

Examples of actual and potential BioMEMS application include: a small device attached onto a contact lens that measures optic pressure and carries out readings on the radio with time, an equipment embedded near the affected site that sends out a required quantity of a drug as needed, and a device less than $1 \mu \mathrm{m}$ in size that can be made to circulate in blood vessels. Although these biosensors and dispatch equipment have progressed rapidly, there is still the problem of devising an effective power supply source. Several proposed possibilities that have been proposed include electric impulses that drive the function of cell membranes, substances such as ATP as sources of energy storage, or blood flow that provides ongoing dynamic energy, etc.[1] (Fig. 11).

All these devices consist of a biosensor using semiconductors. The two types of biosensors considered are: a biomaterial sensor made of a biological substance, and an artificial biomimetic sensor that imitates a part of the living body[12].

1) Biomaterial sensors: This type of sensor uses a biological substance as a detector (receptor). The representative substance is an excellent receptor for specificity and sensitivity.

The basic structure of biomaterial sensors consists of a receptor (acceptance and conversion of a signal), enzyme fixation plate, a selective ion-permeable membrane (generated ion, an oxide, etc.), and a transducer (conversion into electricity). An ionsensitive field effect transistor (ISFET) used for a transducer is a field effect type transistor (FET) which has the surface covered with an ion-penetrating membrane (Fig. 12). This transducer detects a surface potential generated by an ion that penetrates the membrane and adsorbs on the surface[13].

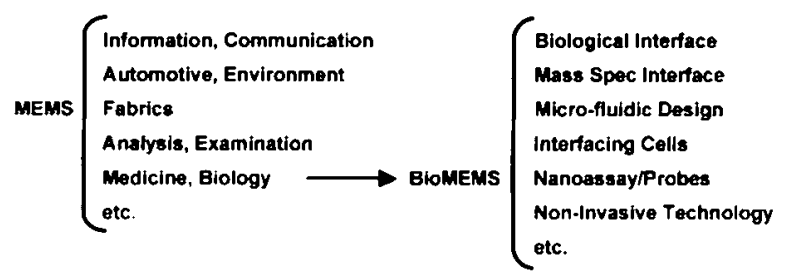

Fig. 11 Mems and biomems
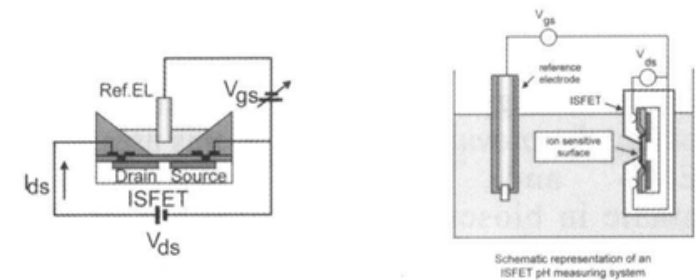

Fig. 12 Isfet (ion-senstive field effect transistor)

For example, changes in ion concentration produced as a result of an intended substance for measurement (substrate) reacting with the enzyme fixed membrane gives potential change through an ionsensitive surface.

With this device, the output current (drain current) as a big amount of change can be taken out corresponding to a minute change of gate voltage, it can be made with high sensitivity. Since a minute ion change (gate voltagc) is amplified and converted into a big output current (drain current), this device can be used as a highly sensitive biosensor. Changing the kind of ion-permeable membranes can raise the ion selectivity.

For example, in a $\mathrm{pH}$ sensor covered with a thin glass layer, the chemical property of a hydrogen ion that penetrates the glass layer is used. If an antibody is fixed on the surface of a gate electrode, a FET device can detect the electric charge given by an antigen that binds the antibody.

Potential candidates for molecule recognition elements in biosensors include enzymes, microbes, antigens, antibodies, genes, cells, organs, tissues, oligosaccharides, lipid membranes, and artificial materials. These elements are used for various types of biosensors according to different detection targets, and not all biosensors necessarily have the ISFET structure. Needless to say, the use of semiconductors and IC technology can bring about many benefits, such as mass production, miniaturization, addition of multiple functions, and cost reduction [14].

In the future, as medical systems become fully integrated with semiconductors, we can envision such technological wonders as biochips that measure information on ions, cell count, or DNA-RNA from a very small quantity of blood or specific cells sampled by a non-invasive needle; or tips to transmit and receive real-time information from sensors located inside the human body[15].

2) Biomimetic sensors: This type of sensor is made of artificial materials, and can mimic or substitute physiological entities or functions. For example, very small tips consisting of many semiconductor elements can be used to investigate artificial retinas or artificial cochleas, etc. 
"MEMS" and "BioMEMS" are already becoming terms of the past due to rapid developments in nanotechnology. And since nanotechnology is fast becoming the prevailing science in this area, the terms "NEMS" and "BioNEMS" will continue to dominate in bioscientific applications of the near future.

\section{BEYOND THE SILICON SEMICONDUCTOR[16-17]}

As for present technology, the miniaturization of semiconductor elements is carried out through topdown processes, i.e., cutting silicon crystals in a thin slice followed by application of lithography using far ultraviolet radiation to print electronic circuitry, and finally dissolution and removal of unneeded portions with chemicals, etc. While this top-down process is sufficient for the present, the technology will soon be confronted with problems as described below (Table $3)$.

* If the line width (gate length) of a circuit becomes as thin as about $10 \mathrm{~nm}$, the variation in IC production exceeds a permissible limit, causing the IC to stop functioning normally.

* Within a silicon semiconductor, drift conductions of electrons cannot be avoided, limiting the speed of electron movement to only $100 \mathrm{~km} / \mathrm{sec}$.

* If the diameter of copper wires used for interconnection becomes as thin as $1 \mu \mathrm{m}$, the wires will break down from heat when the current reaches about $100 \mathrm{~mA}$.

In order to advance miniaturization and to raise the reaction speed of electrons, it is necessary to find a new element other than silicon. One promising candidate is a carbon nanotube. By controlling the structure of the carbon nanotube, its electronic characteristics can be varied from metallic to semiconducting.

The tube consists of a very fine line structure in which carbon atoms have bonded so tightly with each other that disconnection hardly occurs, even at large currents. Since nanotubes have the best thermal conductivity among all substances, they relieve the problems of heat generation in LSI circuits.

Furthermore, in carbon nanotubes, electrons and holes are free from the drift conduction accompanied by a dispersion phenomenon, and are able to move by ballistic conduction. If this is realized, the electrons and holes will be able move at speeds of as high as $500-800 \mathrm{~km} / \mathrm{sec}$ (Fig. 13).
Table 3 Problems and expected technology

\begin{tabular}{|c|c|c|}
\hline Present Technology & Problems & Expected Technology \\
\hline Llthography & Variation & Auto-asesembly \\
\hline $\begin{array}{c}\text { Conduction Velocity } \\
\text { of Electron }\end{array}$ & $\begin{array}{c}\text { Drift Conduction } \\
(100 \mathrm{~km} / \mathrm{sec})\end{array}$ & $\begin{array}{c}\text { Bollistic Conduction } \\
\text { (Z600km/sec) }\end{array}$ \\
\hline Lead Wire (Cu) & $\begin{array}{c}\text { Resistance, Heat, } \\
\text { Disconnection }\end{array}$ & $\begin{array}{c}\text { Now Materials } \\
\text { (Carbon Nanotube, etc.) }\end{array}$ \\
\hline
\end{tabular}

In this state, the electrons will achieve a quantum behavior. Therefore, the realization of a "quantum effect device" such as a "single electron transistor" using the nanotube can be expected. A device with structure fundamentally similar to the conventional MOSFET has been proposed, that is, a source electrode and a drain electrode was molded with a catalyst metal on the insulated film (SiO) of a silicon plate, and a nanotube bridge was constructed between these electrodes by the chemical vapor deposition method (CVD) (Fig. 14).

Although the technology for miniaturization of MOSFETs has been advancing, about tens of thousands of electrons are still required for current passing between the two electrodes. Therefore, the passing speed limit of electrons can be increased under the influence of the aforementioned drift conduction caused by diffusion of electrons.

On the other hand, if a carbon nanotube becomes applicable, only one electron will be able to move inside the tube at a time. If no voltage is applied to the gate electrode, a phenomenon called "coulomb blockade" in which each electron interacts with repulsive force will prevent current conduction. However, if a positive voltage is applied to the gate, the electron will move and induce current flow. Furthermore, ballistic conduction will become possible since drift conduction does not occur in this condition. Elements that function with the movement of one electron at a time are called Single Electron Transistors (SET).

Indeed, the development of semiconductor tips has already improved down to the level of nanoscience, but if their miniaturization advances even further, quantum mechanics will dominate and

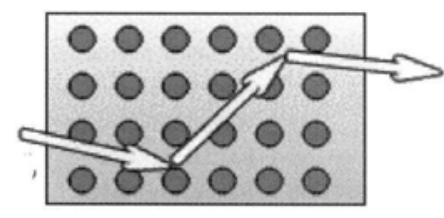

Drift Current

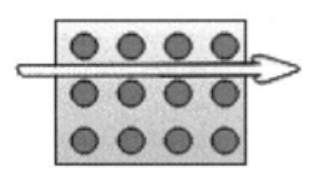

Ballistic Current
Fig. 13 Drift and ballistic conduction of electrons 


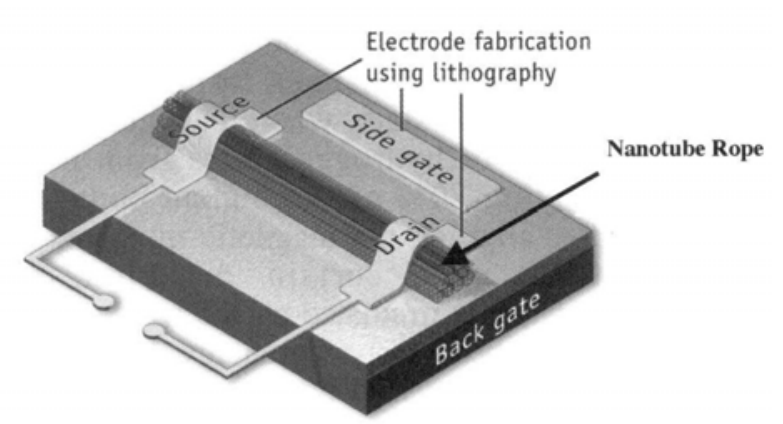

http://www.research.ibm.com/resources/press/Transistors/FE T_array-process.jpg

\section{Fig. 14 Carbon Nanotube Semiconductor}

electrons as particles will acquire the property of waves, and any devices assembled with conventional concepts of electron particles will stop functioning altogether. For example, if the width of a transistor gate becomes extremely narrow, a tunnel phenomenon that brings about insulation failure will arise in the world of quantum electronics. However, considering the needs for furthering the miniaturization of semiconductors, we cannot avoid the realm of quantum electronics. We are approaching a critical juncture and must be prepared to confront the challenges of the quantum realm.

For example, researchers have recently proposed a new idea of using electron spin to create two magnetic moments, "upward/downward." In current semiconductor systems, information processing is conducted by setting electric charges of " $+/-$ " to correspond to " $1 / 0$," and each electron spin has been ignored in functioning because a large number of electrons contribute current flow from the source to the drain. However, the new research field of "spintronics" has been developed in which the property of electron spinning is effectively used for processing information. Although spintransistors (spin FET) used in spintronics has the same form as ordinary FETs, it differs in that the source and the drain are made from a ferromagnetic material. If no voltage is applied to the gate, the electron magnetic moments of the source and drain are the same, allowing current to flow across both sides. On the other hand, if a positive voltage is applied to the gate, differences in the magnetic moment of each electron moving between both ferromagnetic materials will develop, controlling current flow toward the drain. Spintransistors have the advantages of being able to change magnetic moments with little energy and of increasing the speed of electron movements. This concept will ultimately be useful for realizing a quantum computer.

The use of electron spin is not a subject of future research, but one that is already being used every day. That is, the concept of spinning magnetic bodies has been used in operating common hard disk memory devices since the early stages of the personal computer. This device functions by reading resistance changes accompanying magnetic changes. In present designs of hard disks, a giant magneto-resistance (GMR) head has been devised to read high-volume data, yielding large storage capacities of as large as $1 \mathrm{~GB}$ in common personal computers. Other future elements beyond the silicon semiconductor are being investigated, including single molecular devices, plastic semiconductors, and bio-semiconductors.

A typical example of the bio-semiconductor is the DNA computer that was created by Leonard Adleman in 1994[18]. This type of computer is said to be highly efficient in solving high-density information with parallel processing, which are tasks that conventional Von Neumann type computers cannot perform. As is often said, "Silicon is too slow!" for this type of processing (Table 4).

\section{SUPERCONDUCTORS BIOSCIENCE[19-20]}

In 1911, scientists discovered a phenomenon called "superconductivity" where mercury lost its electric resistance almost entirely when cooled to $4 \mathrm{~K}$ absolute degree of liquid helium. However, the underlying principle of superconductivity was not solved for another 50 years until the Bardeen, Cooper, Schriefer (BCS) theory was proposed in the 1970s. Superconductivity has the following six outstanding characteristics (Table 5):

1. Zero resistance makes permanent current possible.

2. Increase of a critical current value brings about generation of a strong magnetic field without power consumption.

3. If the strength of an external magnetic field is below a critical level, the density of magnetic

Table 4 Beyond Silicon Semiconductor

Spintronics: Single Electron Transistor

Molecular Electronics: Single Molecular Transistor, Plastic Transistor

DNA Computer

Superconductor Transistor 
flux in a superconductor is zero (Meissner effect) $\rightarrow$ Magnetic shielding.

4. In second class superconductors that can exist above a critical magnetic level, the density of magnetic flux is not zero $\rightarrow$ Birth of a superconducting magnet (SCM).

5. Digitization of magnetic flux (quantized magnetic flux: fluxoid) in second class superconductors may enable the development of a quantum computer that requires no power consumption and generates no heat.

6. Application of the Josephson effect, in which current flows within the insulator inserted between two superconductors (electron tunneling), may enable the development of an advanced semiconductor.

Due to the impracticality of using expensive liquid helium as cooling substances to obtain superconductivity, researchers are presently competing to discover or invent high-temperature superconductors such as oxide superconductors and fullerene superconductors, which function at temperatures above liquid helium conditions. Among the substances reported so far, $\mathrm{Hg}_{2} \mathrm{Ba}_{2} \mathrm{Ca}_{2} \mathrm{Cu}_{3} \mathrm{O}_{\mathrm{x}}$ functions as a superconductor at the highest temperature $(135 \mathrm{~K})$. For practical purposes, however, developers are focusing on superconductors that can be synthesized with simplicity.

In Japan, it was discovered that magnesium boride $\left(\mathrm{Mg}_{2} \mathrm{~B}\right)$ substances consisting of only two kinds of elements behaved as a superconductor even at the low temperature of $39 \mathrm{~K}$. The development history of hightemperature superconductors is shown in Fig. 15.

At present, the functions of commercially available superconducting devices have improved drastically through the application of superconductive magnets. Examples of these devices include nuclear magnetic resonance equipment (NMR) used for the analysis of chemical structures, and magnetic resonance imaging (MRI) systems used for medical diagnosis.

\section{Table 5 Six Major Characteristics of Superconductivity}

Six Major Characteristics of Superconductivity

1. No resistance, permanent current

2. Strong magnetic field without power consumption

3. Magnetic shielding (Meissner effect)

4. Superconducting magnet (SCM)

5. A quantum computer using fluxoid

6. An advanced semiconductor with Josephson junction device
The principle of a strong superconducting magnetic field aligning the direction of electron spins in the same direction has been applied to the superconducting MRI system, where changes in the magnetic moments of hydrogen atoms from body moisture occupying $70 \%$ of the human body are measured. After aligning the magnetic moments of hydrogen atoms in a certain direction, an electromagnetic wave is irradiated on the whole body. The degree of absorbed resonance energy emitting is then measured and transferred into image processing (Fig. 16).

By virtue of this system, it has become possible to perform non-invasive diagnosis of physiological and pathological conditions of internal soft tissue without interference from bone tissue. Furthermore, since the frequency of irradiated electromagnetic waves is similar to that of radio waves, there is no fear of harmful side effects to the living body caused by X-ray or other types of strong radiation.

The stronger the magnetic field is applied, the better the hydrogen magnetic moments is aligned, yielding more sensitive diagnostic imaging with superconducting MRI systems.

According to quantum theory, light that shows wave motion acquires the characteristics of particles (photons), and electrons as particles take on the features of waves (material waves). A superconductor in which electrons serve as waves is a Josephson device, representing a macroscopic quantum effect.

In a superconductivity state, a feeble magnetic field can be detected by using the interference between electron waves and a magnetic field. A device

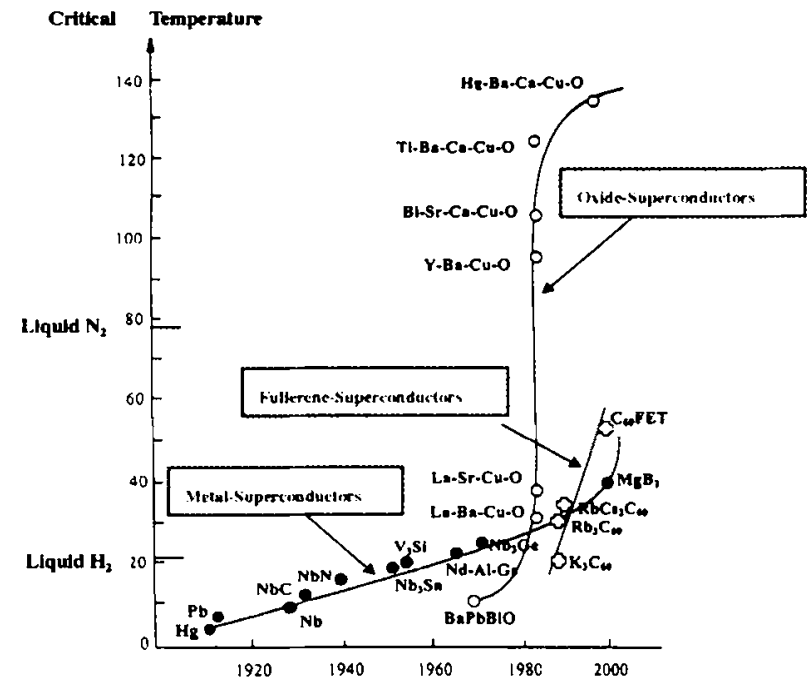

Fig. 15 Development of High-Temperature Superconductors 
applying this principle is the Superconducting Quantum Interference Device (SQUID), enabling real time measurements of magnetic field intensity that cannot be performed by MRI. For example, SQUID can detect fecble magnetic field changes induced by dynamic neuronal currents, and is suitable for diagnosing cardiac functions and measuring fetal development.

When a feeble magnetic flux generated by bioelectric currents passes through a superconductivity circuit containing one or two weak Josephson junctions, a measurable current signal corresponding to the passage of the magnetic flux is generated.

Since the magnetic field generated by bioelectric currents is very fecble, surrounding magnetic fields including geomagnetic disturbances have to be eliminated when operating SQUID. By virtue of the Meissner effect, an inherent characteristic of superconductors, any weak external magnetic ficld cannot disturb SQUID measurements. Therefore, SQUID uses superconductors in the following two functions: the detection part consisting of the Josephson devices, and the magnetic shicld attained by the Meissner effect (Fig. 17).

Computers packing more processing power onto the tips of silicon semiconductors are indispensable for advancing research in the life sciences.

For the near future of silicone semiconductors, Moore' $s$ law predicts that limitations on increased processing speed and capacity are inevitable. Recent

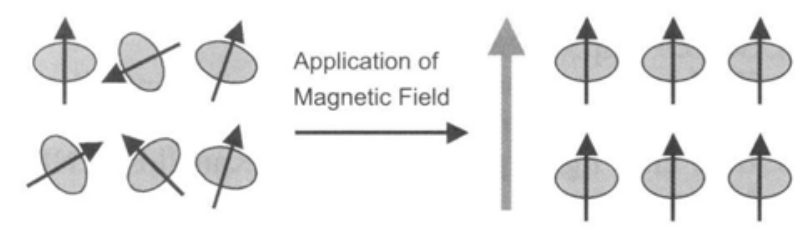

Fig. 16 Alignment of Magnetic Moments of Electrons under Strong Magnetic Field

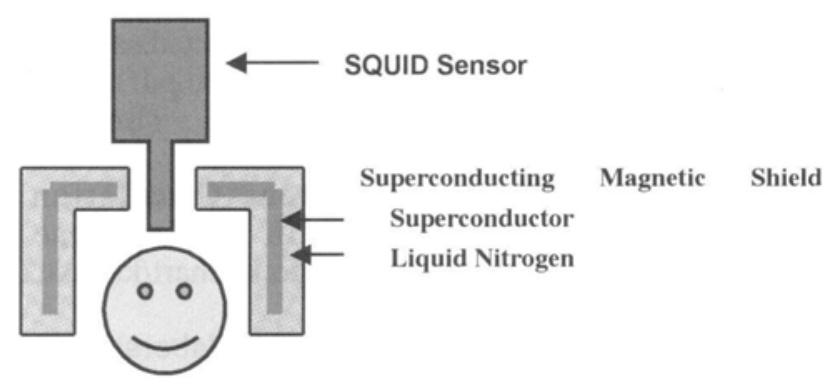

Fig. 17 SQUID System for Measuring Brain Activity developments in technology for increasing information volume and processing speed have caused adverse problems such as increasing power consumption and heat generation. If superconductivity devices are introduced, these problems accompanying high-density accumulation of silicone semiconductors can be resolved.

By using the tunnel effect generated in a Josephson device, an ON/OFF switch that is the fundamental function of a computer principle is obtained. That is, when the device behaves as a superconductor, a very slight current flows through an insulator ( $\mathrm{ON}$-function); alternatively, when a current intensity imposed on the Josephson device exceeds a certain level, the device will lose superconductivity and the tunnel effect will disappear (OFF-function).

In recent research, a superconductivity computer using a digitized magnetic field by superconductors is being investigated. This computer uses an SFQ circuit (single flux quantum) in which the presence or absence of the minimum unit of SFQ in a ring-shaped superconductor corresponds to a "l "or "0." Since a superconductor repels magnetic flux, a part of the Josephson junction is inserted into a ring-shaped superconductor so that the SFQ enables entry into the ring. As compared with a semiconductor, it is presumed that the switching speed of an SFQ device is 100 times faster, and power consumption is only $1 / 100$ that of semiconductors, transmitting in a superconductive wire at speeds near the velocity of light.

If the high temperature superconductors described above can be used freely, groundbreaking progress and developments in the life sciences within this century will be highly probable in the fields of sample analysis, diagnosis, medical treatment, etc.

\section{BEYOND ELECTRONICS}

Scientists are devoting every effort to apply nanotechnology or superconductor technology in order to resolve the difficult problems that surround silicon semiconductor technology. Even if these efforts are taken to the highest possible levels, the technology still remains within the limits of electronics. To exceed this limitation, the next level of research on semiconductors must break away from silicon. Photonics, a new field of research focusing on manipulating photons instead of electrons, has already started.

\section{CONCLUSION}

The ongoing developments in semiconductors and 
superconductivity for bioscientific applications were outlined in this report.

It is well known that germanium (Ge) and silicon (Si) are elements with electrical conductivities that are intermediate between those of conductors and insulators. The semiconducting property of $\mathrm{Si}$ was remarkably improved by incorporating a foreign atom, such as phosphorus, arsenic, antimony, or boron into the crystal lattice of a silicon atom. This improvement led to the creation of diodes, bipolar-semiconductors, field effective semiconductors, and finally to the invention of integrated circuits (IC) through advanced miniaturization techniques. Thus, advanced semiconductor technology has been applied towards inventing various minute machine systems called Micro Electro-Mechanical Systems (MEMS), including the system relating to medicine and biology (Bio MEMS). Bio MEMS incorporates electronic circuits, chemical reaction cells, a control system, an analysis apparatus and machine parts onto a thin silicon tip, and is applied to extra and intracorporeal uses.

The progress of IC technology has so far fulfilled the 1965 prediction of "Moore' s law" stating "the number of transistors on a given chip can be doubled every two years", but the law may not be applicable by the year 2010 due to various limitations on existing technology. For instance, transistors still retain the same structure and principle as that of the first MOSFET invented in 1974, were the distance from a source to a drain, that is a gate length, serves as a limiting factor in miniaturization of transistors. If miniaturization continues along the MOSFET principle, a number of technological problems, such as current leakage, generation of heat, and disconnection may occur. Therefore, a new science exceeding the capabilities of present silicon semiconductors is needed. Spintransistors, single-molecular transistors, bio-transistors, and superconductor transistors are leading candidates that offer processing speeds far superior to silicon.

It is well known that the functions of chemical and medical devices such as nuclear magnetic resonance (NMR) and magnetic resonance imaging (MRI) have improved drastically through the application of superconductive magnets. Furthermore, the Superconducting Quantum Interference Device (SQUID), a device enabling real time measurements of magnetic field intensity that cannot be performed by MRI, is now available for detecting feeble magnetic field changes induced by neuronal currents, diagnosing cardiac functions and measuring fetal development, etc.

Researchers are currently investigating a superconductivity computer that uses a digitized magnetic field by superconductors using a single flux quantum (SFQ) circuit. It is presumed that the switching speed of an SFQ device is 100 times faster, and power consumption is only $1 / 100$ that of semiconductors, transmitting in a superconductive wire at speeds near the velocity of light.

Scientists are devoting every effort to apply nanotechnology or superconductor technology in order to resolve the difficult problems that surround silicon semiconductor technology, and the next level of research on semiconductors must break away from electronics. In particular, scientists are already researching the new field of photonics, which is primarily concerned with manipulating photons instead of electrons.

\section{REFERENCES}

1. Nakamura, S: BioMEMS that changes therapy and science. Nikkei Science. 1, Dec 2000 (in Japanese)

2. BioNEMS Symposium, May 22, 2004, USC, Calif. USA

3. Nishikubo Y: Semiconductor. Visual guide book. Shuwa System. Tokyo. 2003 (in Japanese)

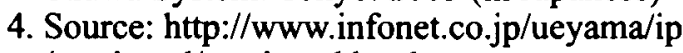
/semi_cnd/semi_cnd.html

5. Source: http://www.infonet.co.jp/ueyama/ip/ glossary/semi_cnd_p.html

6. Source: http: $/ / \mathrm{www}$.infonet.co.jp/ueyama/ip/ glossary/semi cnd_n.html

7. Source: $\mathrm{http}: / / \mathrm{ww}$.intel.com/research/ silicon/mooreslaw.htm

8. Source: http://public.itrs.net/

9. IBM announces world' $s$ smallest working silicon transistor. IBM Press Releases, Dec 9, 2002

10. IBM chip fights power leaks. M. Kanellos. CNET News. com. Sep 9, 2002 (source: h t t p://zdnet.com .com/2100-1103 957087.html?tag=nl)

11. Tri-gate transistor moving from research to development.

(source: http://www.intel.com/update/contents/si07031.htm)

12. Hara M. Sensors -from chemical sensors to biosensors - (in Japanese) (http://www.tsd.saitamau.ac.jp/ nagai/group/H1004/pdf/genkou.pdf)

13. ISFET Technology (Source: http://www.sentron.nl/)

14. Supporting Theme for Patent Circulation: Biosensors, 2001. (in Japanese) (Source:http://www.ryutu.ncipi.go.jp/chart/kagaku2 /1/1-1.htm)

15. Expectation to and problems of collaboration of biology and nanotechnology. May 24, 2002 (in Japanese)(Source:http://www.fed.or.jp)

16. Awano Y: Go beyond the limit of silicon. Nikkei Science. 32-37, Aug 2002 (in Japanese) 
17. Spintronics. Nikkei Science. 16-25, Sept 2002 (in Japanese) (original article: Awschalom DD et al. Spintronics. Scientific American June 2002)

18. Adlemen LM. Molecular computation of solutions to combinatorial problems. Science 266: 102 1994. (Source:http://www.nanoelectronics.jp/ kaitai/dnacom/index.htm)

19. Murakami M. A new era of superconductivity. $\mathrm{K}$ Book Series 166, Kogyo Chosakai. 2001 (in Japanese)

20. Condensed matter physics. From microphysics to macrophysical properties. Ed. by Masumi T, Nikkei Science Supplement p121, 1997 (in Japanese) 